\title{
An improved biomechanical testing protocol for evaluating spinal arthroplasty and motion preservation devices in a multilevel human cadaveric cervical model
}

\author{
Denis J. DiAngelo, Ph.D., and Kevin T. Foley, M.D. \\ Departments of Biomedical Engineering and Neurosurgery, The University of Tennessee Health \\ Science Center, Memphis, Tennessee
}

\begin{abstract}
Object. An experimental study was performed to determine the biomechanical end-mounting configurations that replicate in vivo physiological motion of the cervical spine in a multiple-level human cadaveric model. The vertebral motion response for the modified testing protocol was compared to in vivo motion data and traditional pure-moment testing methods.

Methods. Biomechanical tests were performed on fresh human cadaveric cervical spines (C2-T1) mounted in a programmable testing apparatus. Three different end-mounting conditions were studied: pinned-pinned, pinned-fixed, and translational/pinned-fixed. The motion response of the individual segmental vertebral rotations was statistically compared using one-way analysis of variance and Student-Newman-Keuls tests $(\mathrm{p}<0.05$ unless otherwise stated) to determine differences in the motion responses for different testing methods.

Conclusions. A translational/pinned-fixed mounting configuration induced a bending-moment distribution across the cervical spine, resulting in a motion response that closely matched the in vivo case. In contrast, application of puremoment loading did not reproduce the physiological response and is less suitable for studying disc arthroplasty and nonfusion devices.
\end{abstract}

\section{KEY WORDS - biomechanical testing protocol $\bullet$ cervical spine $\bullet$ biomechanics $\bullet$ disc arthroplasty - motion segment unit kinematics}

Spinal instrumentation is commonly used to provide immediate stabilization and to promote anterior cervical fusion. Unfortunately, fusion surgery has been reported to increase the biomechanical stresses at the adjacent segments, which could lead to further degenerative symptomatic disc disease at the adjacent levels. ${ }^{4,6}$ An alternative approach to cervical fusion surgery is to restore motion to the diseased segment with disc arthroplasty. The goal of the disc prosthesis is to replace the diseased disc while preserving and/or restoring the motion at the treated spinal level. Limited biomechanical data exist for analysis of the effects of disc arthroplasty on cervical spine biomechanics; the most suitable method for evaluating these mobile spinal devices in vitro remains unclear.

Biomechanical testing on human cadaveric tissue offers a practical means for evaluation and ranking of different surgical techniques; however, there are no standard tissue-based testing protocols for evaluating spinal devices. Furthermore, in vitro tests can be performed under load control, displacement control, or more recently, hybrid control (combinations of load and displacement) through the use of multiple-axis, robotic controllers. ${ }^{10,12,16,17,18,36}$

The cervical spine consists of a series of free VBs that

Abbreviations used in this paper: IAR = instantaneous axis of rotation; $\mathrm{MSU}=$ motion segment unit; $\mathrm{PF}=$ pinned-fixed; $\mathrm{PP}=$ pinned-pinned; $\mathrm{TPF}=$ translational/pinned-fixed; $\mathrm{VB}=$ vertebral body. exhibit complex, coupled motions and loading behaviors. For the subaxial cervical spine, in vivo motion is greatest in the sagittal plane, with more rotation occurring in extension than in flexion. ${ }^{35}$ Only small amounts of muscle activity are needed to maintain the head's orientation in an erect (neutral) position. Thus, muscle-induced compression is small and head weight is the typical physiological force that acts on the cervical spine. Flexion or extension of the head induces a bending-moment distribution throughout the cervical spine that increases caudally and acts in combination with the compressive (head weight) force (Fig. 1). In vitro testing methods should replicate this motion response.

The objective of this study was to identify the appropriate loading conditions that would replicate the in vivo motion response of the cervical spine, and thus to develop a biomechanical testing protocol for evaluation of disc arthroplasty or motion preservation devices. The motion response for the improved testing method was compared with in vivo motion data obtained from the literature and with traditional in vitro pure-moment methods.

\section{MATERIALS AND METHODS}

\section{Biomechanical Testing Apparatus}

A programmable, single-actuator, biomechanical testing apparatus was used in this study., ${ }^{3,79}$ The rigid frame 


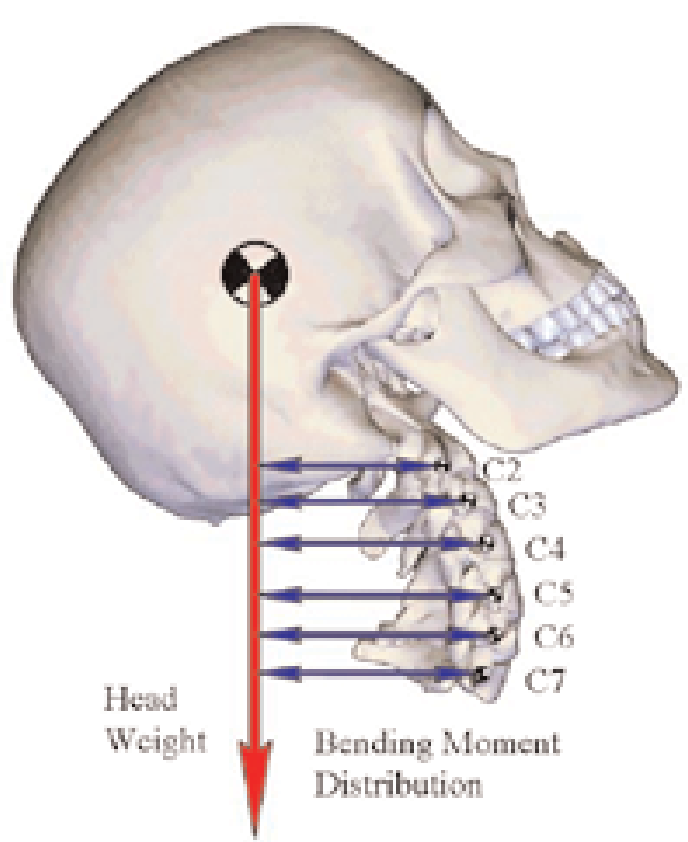

Fig. 1. Computer model showing in vivo sagittal motion and mechanics of the cervical spine. This illustration depicts the caudally increasing bending-moment distribution $(2-5 \mathrm{Nm})$, which is greatest at the C5-6 level, and is created by continuous rotations with like polarity at each vertebral level ( $40^{\circ}$ flexion or extension) with an axial compressive load (50-N head weight).

housed a servomotor load actuator (Industrial Device Corp., Novato, CA) connected to a robotic controller (Adept, Inc., San Jose, CA). A single-axis load cell (Transducer Technologies, Temecula, CA) was inline with the shaft of the load actuator. The other end of the singleaxis load cell was coupled to end mounting fixtures that regulated the end motion and loads applied to the spinal construct. A six-axis force sensor (JR3, Inc., Woodland, CA) was rigidly affixed to the base of the test frame to which the opposite end of the spine was rigidly fixed and attached. The multiple-axis load cell reported in real time the three orthogonal forces and moments that were transferred through the spine.

\section{End Mounting Configurations}

A sequence of tests had been previously performed to analyze different mounting configurations and appropriate ranges of load and motion to identify a particular set of end conditions that produce normal motion of the intact spine tissue. ${ }^{7}$ The gold standard of motion was replication of positions considered normal by anatomists, physical therapists, and spine surgeons (Fig. 2). Three different mounting configurations were evaluated: $\mathrm{PP}, \mathrm{PF}$, and TPF. For the first two conditions, the lower mounting fixtures were unclamped and free to rotate or they were fixed. The upper mounting fixtures converted the single controlled input from the load actuator to a rotational input alone or a coupled-motion input (unconstrained translations and/or rotation in the sagittal plane) and a combined loading state (axial compressive force alone or with a flexion/extension bending moment).
For the TPF configuration, the upper fixture consisted of a linear bearing and splined shaft assembly that mounted in a rotating joint attached to the actuator (Fig. 3). The linear bearing provided a virtually frictionless method for the splined shaft to move relative to the actuator, thus minimizing the shearing forces. The flexion/extension axis of the spine was placed eccentric to the load axis of the actuator and induced a compressive load and flexion/extension bending moment to the upper pot. The specimens were mounted in an inverted neutral orientation with the $\mathrm{T}-1$ pot attached to the upper fixture and the C-2 pot mounted to the lower base fixture, thereby inducing a greater moment at T-1 than at C-2. Specimens with gross alignment deformities were not used.

A rotational displacement transducer (Data Instruments, Acton, MA) was attached to the rotational joint connected to the actuator; this transducer measured the global rotation of the spine. A translational displacement transducer (Data Instruments) was inserted into a customdesigned plate between the upper pot and splined shaft connection; the translational displacement transducer measured changes in the moment arm length. For lateral bending tests, the spine was rotated $90^{\circ}$ in the mounting fixtures and the base was unconstrained in axial rotation. A separate loading system was used for axial rotation. The testing arrangements for lateral bending and axial rotation are shown in Figs. 4 and 5.

\section{Nondestructive Testing Protocol}

All tests were performed under displacement control with the subaxial (C2-T1) cervical spine positioned either inline (PP and PF conditions) or eccentric (TPF condition) to the center line of the vertical actuator shaft. The actuator was programmed to output a triangular displacement/ time waveform of $6.4 \mathrm{~mm} / \mathrm{second}$. For each configuration, an increasing incremental displacement was applied until a target moment between 3 and $4 \mathrm{Nm}$ was reached at $\mathrm{T}-1$, or it was stopped if any of the following limits were reached: $40^{\circ}$ of sagittal plane rotation; $5-\mathrm{Nm}$ bending or torsional moment at T-1; or axial compressive load of 500 $\mathrm{N}$ for the PP and PF configurations or $75 \mathrm{~N}$ for the TPF configuration. These values were based on our preliminary test findings ${ }^{7}$ and were in agreement with the limits used by other researchers. ${ }^{26,28}$ The spines were preconditioned with five cycles before formal testing. Each test trial included three loading cycles; the third cycle was analyzed.

For the PF configuration, minimal displacement of the actuator quickly produced a highly compressive load that exceeded the allowable load limit and induced minimal amounts of vertebral motion. The PP configuration produced an unordered, bipolar motion response similar to that associated with the first mode of column buckling. Furthermore, for both the PP and PF configurations, a nonphysiological internal moment was created in the spine. Nevertheless, with the TPF configuration, all VBs moved continuously with the same polarity of rotation. A distributed moment was applied throughout the cervical spine. Therefore, the TPF configuration satisfied the external loading criteria necessary to replicate the in vivo motion response of the cervical spine. For the purpose of this study, the in vitro results of the MSU patterns of the 


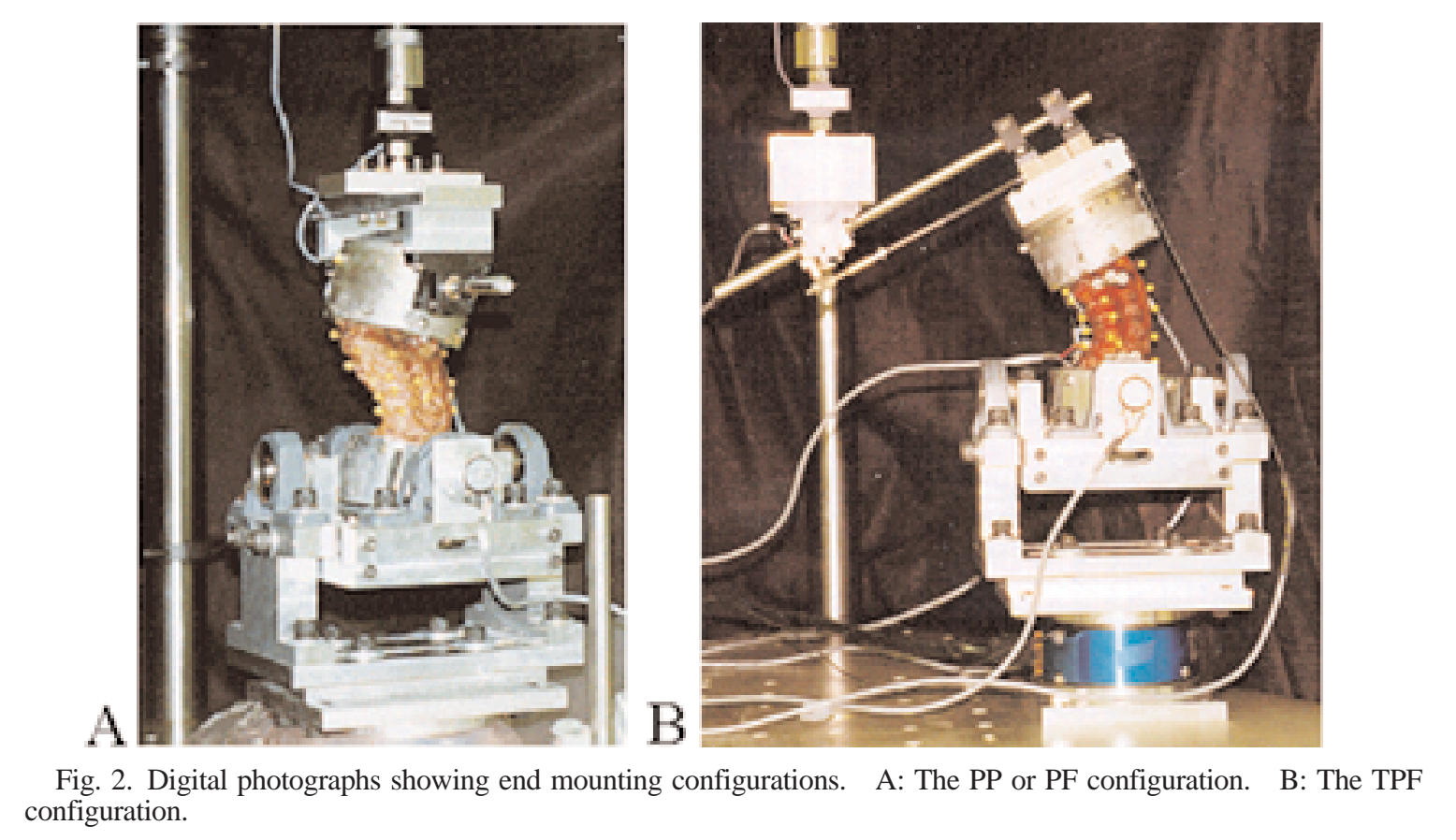

intact harvested spines generated with the modified testing protocol were compared with published in vivo motion data and motion data associated with pure-moment testing methods. ${ }^{1,13,22,25,35}$

\section{Specimen Preparation and Spine Conditions}

Thirty-three fresh human cadaveric cervical spines (C2-T1) were procured from the Medical Education Research Institute (Memphis, TN). The mean age of the harvested spines used was $72.6 \pm 10.8$ years $(24$ spines from men and 16 from women). The spines were harvested and immediately double-wrapped in plastic bags and stored at $-20^{\circ} \mathrm{C}$ until preparation. Before preparation the spines were thawed in a refrigeration system for 12 hours. All spines were screened with anteroposterior and lateral radi- ographs to exclude any with gross osteopenia or anatomical abnormality. Bone density measurements were not done; however, any specimen that did not provide adequate screw purchase, as determined by the spine surgeon (K.T.F.), was not used. All spines were evaluated in the six different modes of loading: flexion, extension, right and left lateral bending, and right and left axial rotation.

Prior to testing, the free ends of the VBs of C-2 and T1 were mounted in cylindrical pots by using an alignment frame that positioned the cervical spine specimen in a neutral (upright) orientation. The flexion/extension axis was estimated at the anterior aspect of the facet joint of each vertebra. Before the formal testing sequence began, each spine was preconditioned with five cycles at low displacement levels. Each test trial included three loading cycles. Throughout the entire testing sequence the spines

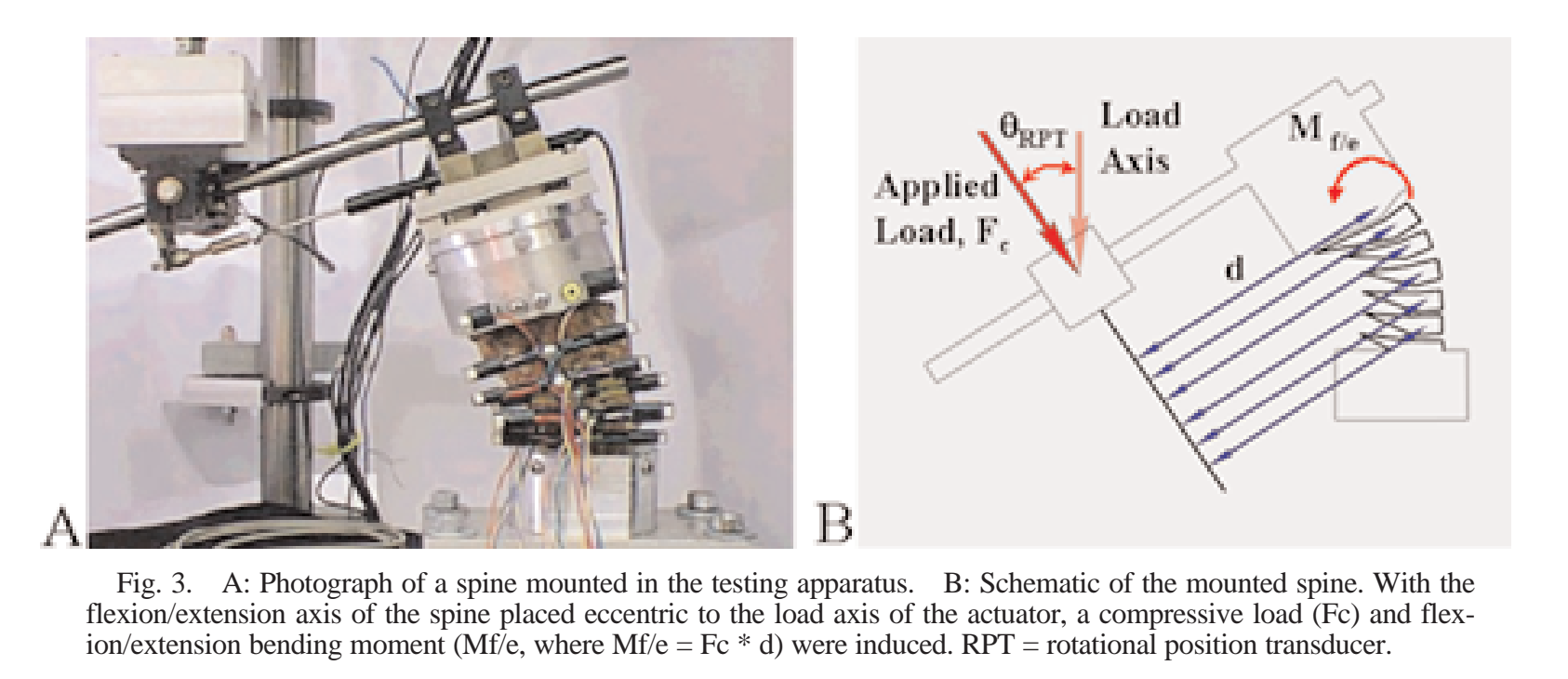




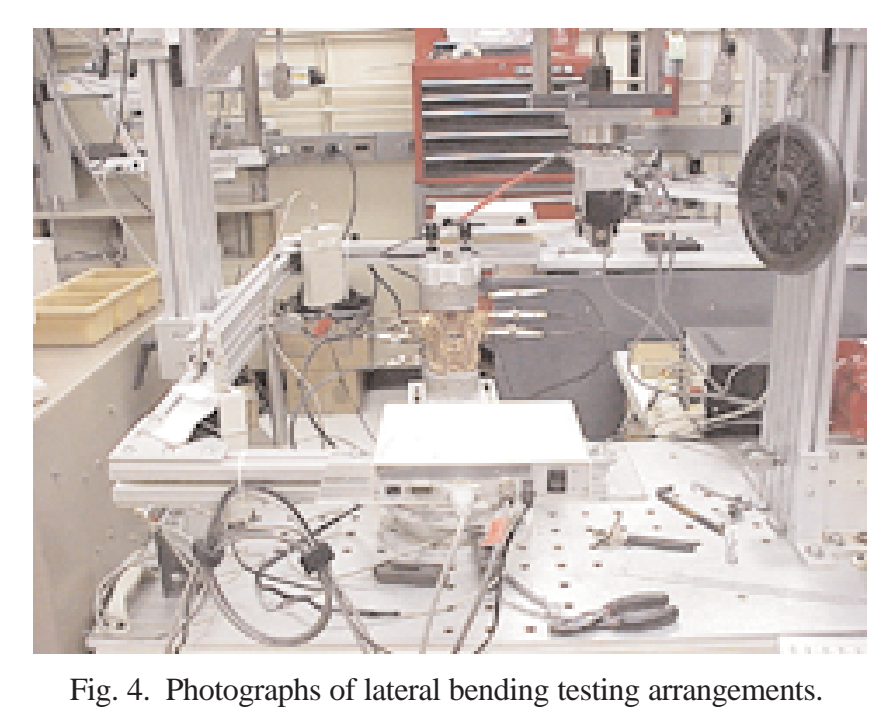

were moistened at regular intervals with a normal saline mist.

\section{Noncontact Motion Measurement System}

A three-dimensional noncontact real-time measurement system was used to track segmental cervical motion for each testing condition. ${ }^{8,10,12,16}$ For the two-dimensional motion analysis, target arrays consisting of two light-emitting diodes were rigidly attached to each spinous process (Fig. 3A). The individual MSU rotations were then expressed relative to the subjacent VB by using principles of rigid body mechanics.

\section{Data Management and Analysis}

For each loading method, the relative change in the individual local MSU rotations was recorded. The individual MSU responses were expressed relative to their percent contribution to the overall global (C2-T1) rotation and then compared. A one-way analysis of variance with Student-Newman-Keuls test $(\mathrm{p}<0.05$ unless otherwise

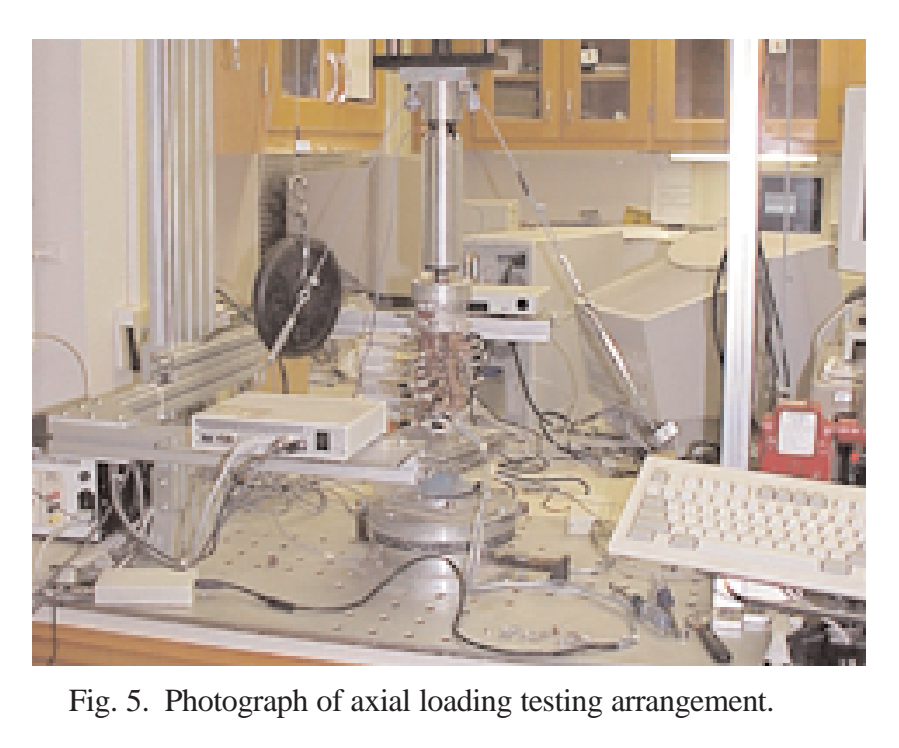

stated) was used to analyze statistically the motion response for the modified testing protocol compared with published data for pure-moment loading methods and with a set of kinematic data from Lysell. ${ }^{23}$

\section{RESULTS}

\section{The Mean Relative Rotations}

The mean relative flexion and extension MSU rotations of the harvested spine corresponding to the modified loading protocol are shown in Fig. 6. The mean in vivo data from the published literature for the flexion/extension MSU rotations were available for MSU segments C2-7 and are plotted in Fig. 7.,1,13,22,35 The corresponding combined flexion/extension MSU rotations for the modified protocol and pure-moment loading method ${ }^{25}$ are also shown in Fig. 7, along with an additional set of flexion/ extension cervical motion data derived by Lysell. ${ }^{23}$ The percent contribution of each MSU rotation expressed relative to the total global rotation for flexion and extension is shown in Fig. 8. The in vitro MSU rotational percent contribution for the modified protocol was similar to that of published in vivo data; ${ }^{1,13,22,35}$ the highest range of flexion/extension motion occurred at the C5-6 MSU, as was observed by Lysell. A significant difference occurred at C2-3 between the modified compared with pure-moment and the Lysell compared with pure-moment method. For pure-moment loading, the rotational response at the lower cervical segments (C4-7) increased slightly in magnitude as the spine moved in the caudal direction. This pattern was the opposite of the response for all other data.

The mean relative MSU rotations for right and left lateral bending with the modified loading protocol are shown in Fig. 9. No in vivo data were available for comparison of individual MSU rotational patterns. The combined right plus left lateral bending MSU rotations for the different in vitro methods are shown in Fig. 10. The percent contribution of each MSU rotation expressed relative to the total global rotation for lateral bending is shown in Fig. 11. The MSU percent contribution of the MSU rotations under lateral bending loads for the modified protocol were comparable to the data published by Lysell. Application of a pure moment induced a significantly greater response at C3-4 and C4-5, which dropped off signifi-

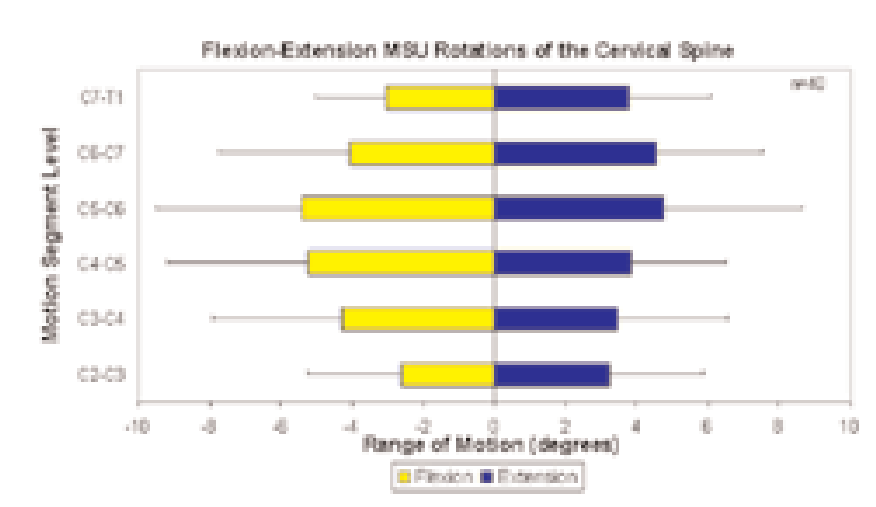

Fig. 6. Bar graph showing flexion/extension MSU rotations of harvested spine for modified testing protocol. $\mathrm{N}=$ number. 


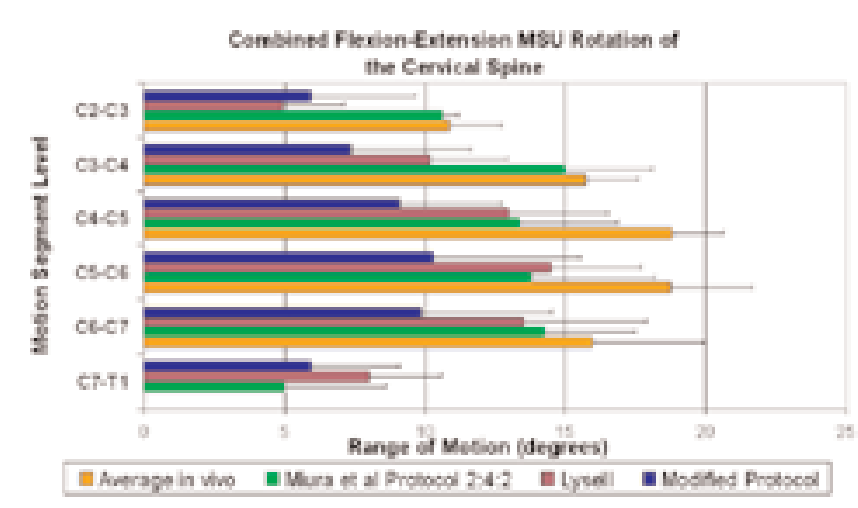

Fig. 7. Bar graph showing combined flexion/extension MSU rotations for in vitro testing methods compared with published in vivo data. The greatest motion occurred at the C5-6 MSU.

cantly across the lower segments. This pattern was not present with the modified method or with Lysell's data. Significant differences occurred at C4-5 between modified compared with pure-moment and Lysell compared with pure-moment methods; at C6-7 between modified compared with pure-moment, modified compared with Lysell, and Lysell compared with pure-moment methods; and at C7-T1 between modified compared with puremoment, modified compared with Lysell, and Lysell compared with pure-moment methods.

The mean relative axial MSU rotations of the harvested spine for the modified loading protocol are shown in Fig. 12. In vivo data from the published literature on the axial MSU rotations were available for MSU segments C2-7 and are plotted in Fig. 13.1,13,22,25,35 The corresponding combined axial MSU rotations for the modified protocol and pure-moment loading method ${ }^{25}$ are also shown in Fig. 13, along with an additional set of axial cervical motion data derived by Lysell. ${ }^{23}$ The percent contribution of each MSU rotation expressed relative to the total global rotation for axial loading is shown in Fig. 14. The in vitro MSU rotational percent contribution for the modified protocol was similar to that reported by Lysell and was comparable to

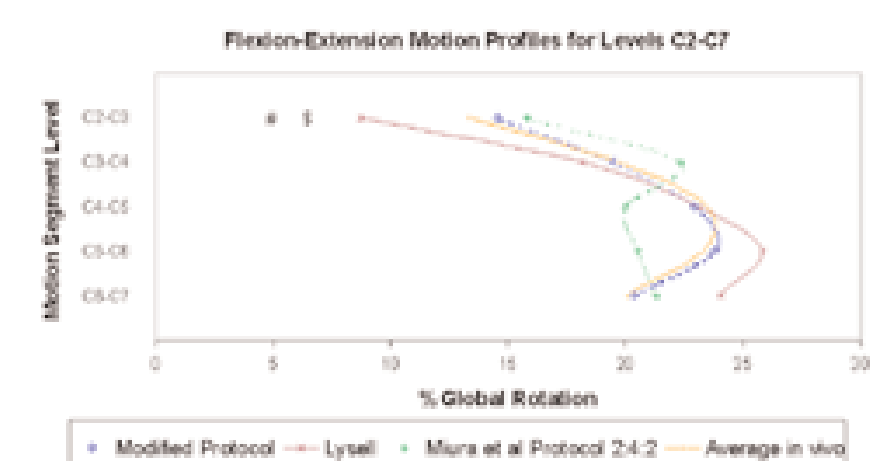

Fig. 8. Graph showing MSU contribution of global flexion/ extension motion for different in vitro testing methods compared with published in vivo data. Pound sign denotes significant difference between modified and Lysell methods. Dollar sign denotes significant difference between pure-moment and Lysell methods.

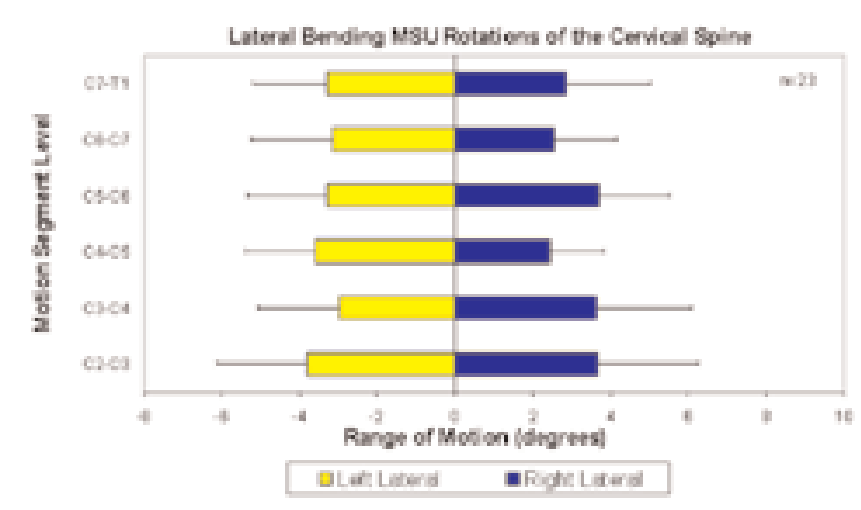

Fig. 9. Bar graph showing lateral bending MSU rotations of harvested spine for modified testing protocol.

published in vivo data. ${ }^{1,13,22,35}$ Significant differences occurred at C6-7 between modified compared with puremoment methods.

\section{DISCUSSION}

\section{Biomechanical Testing Apparatus}

Physiologically relevant studies of tissue-implant mechanics require testing systems that replicate the complex, coupled physiological motions of and loads on human joints. With respect to the cervical spine, a series of free bodies must be analyzed for different motion/load end conditions that are prescribed or controlled by the testing device. The motions of interior spinal bodies are measured, but cannot be controlled.

Simple mechanical devices continue to be used that incrementally apply pure static moments to the spine. ${ }^{24}$ More commonly, however, programmable testing systems are used. Smith, et al., ${ }^{32}$ used an MTS machine to study spine mechanics; the mounted specimen was highly constrained (no motion was permitted above or below the area of interest) and did not replicate physiological motion or loading conditions. Custom fixtures must be added to standard materials testing machines to permit coupled motions, but these machines often remain limited to simple loading scenarios: tension/compression, pure torsion, or four-point bending.

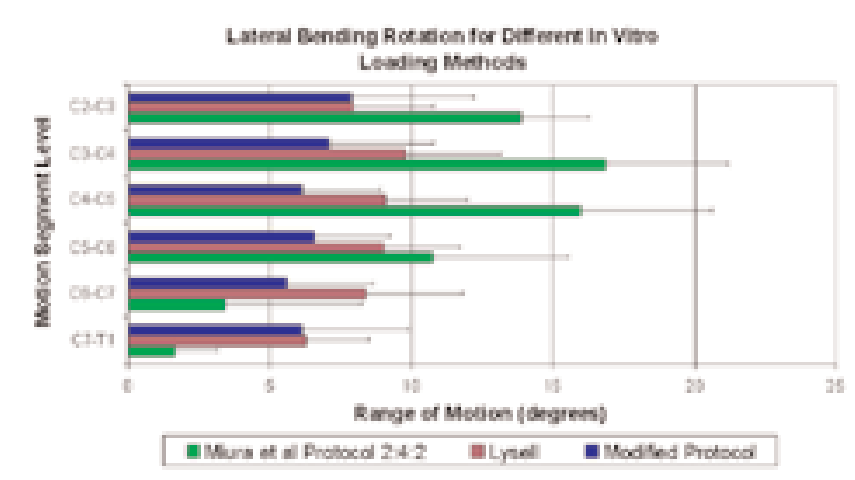

Fig. 10. Bar graph showing combined lateral bending MSU rotations for in vitro testing methods compared with published in vivo data. 


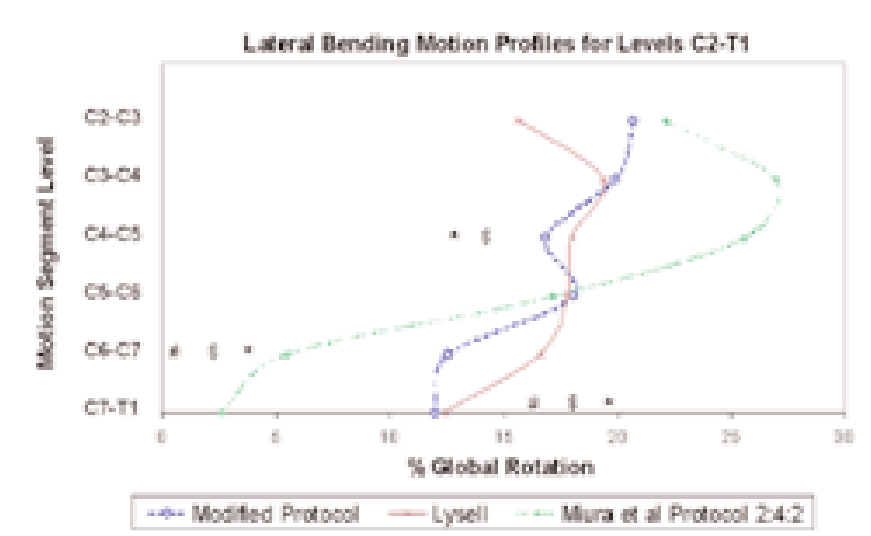

Fig. 11. Graphs showing MSU contribution of global lateral bending for different in vitro testing methods compared with other in vitro loading methods. Asterisk denotes significant difference between modified and pure-moment methods. Pound sign denotes significant difference between modified and Lysell methods. Dollar sign denotes significant difference between pure-moment and Lysell methods.

Weinhoffer, et al., ${ }^{34}$ added a slotted plate fixture to an MTS actuator to enable spinal rotation with nonvertical translation. As the actuator moved down, the upper pot attachment was free to rotate but was constrained to follow a slotted path. The orientation of the slot imposed a specific horizontal as opposed to vertical translational relationship of the upper spinal body that, in turn, was nonphysiological. Kunz, et al., ${ }^{21}$ modified a two-degrees of freedom MTS machine by adding a third rotational degree of freedom to the base of the device. The device was used for pure moment testing with or without an axial compressive load.

James, et al., ${ }^{20}$ presented a two-degrees of freedom spine tester that regulated axial rotation with either flexion/extension or lateral bending. Independent control of each motor prevented force feedback or force limit control features. Shea, et al., ${ }^{31}$ developed a three-degrees of freedom testing apparatus for planar analysis of spinal biomechanics that provided independent control of the displacement output of each axis, but no force feedback control schemes were provided.

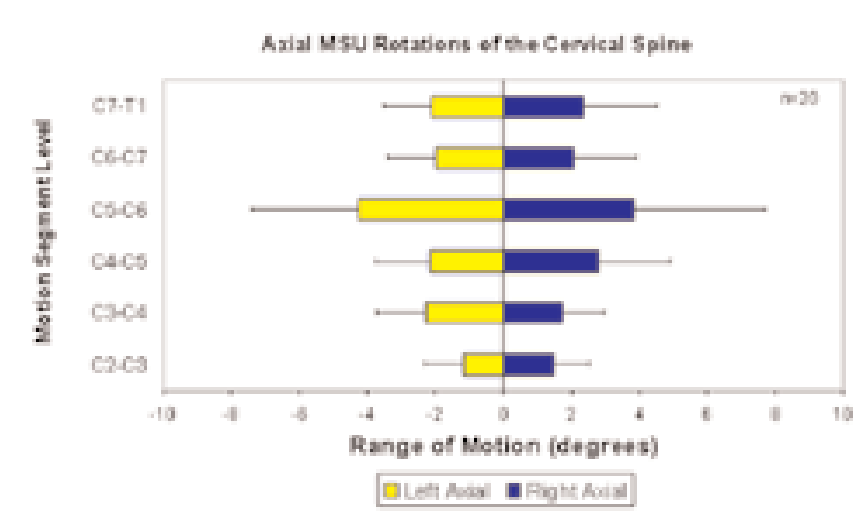

Fig. 12. Bar graph showing axial MSU rotations of harvested spine for the modified testing protocol.

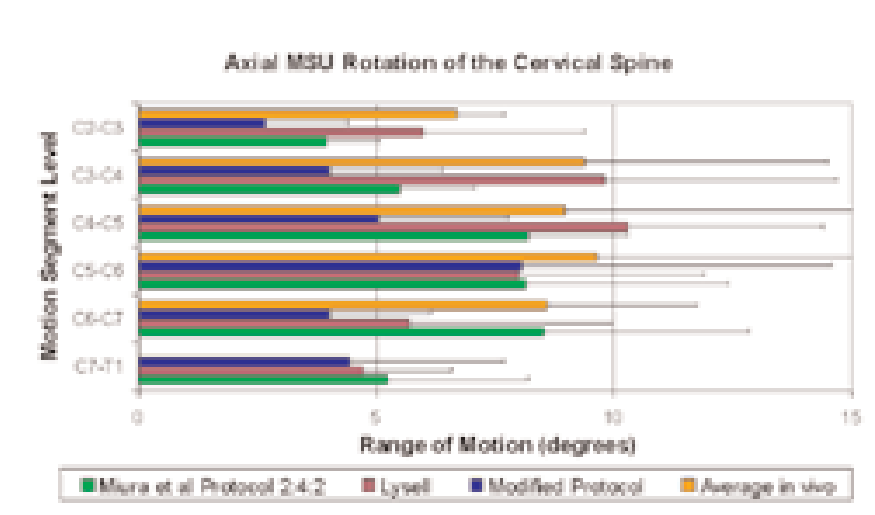

Fig. 13. Bar graph showing combined axial MSU rotations for in vitro testing methods compared with published in vivo data.

Wilke, et al., ${ }^{35}$ developed a spine tester that applied pure moments to the superior end of a spinal construct in three orthogonal directions through the use of counterbalanced stepper motor units attached to the superior end of a spinal construct. The testing protocol was limited to pure-moment loads only. More recently, Gilbertson, et al., ${ }^{17}$ used robotics technology to study single-MSU lumbar spine mechanics. Extensive modifications to the manipulator itself were required (at significant expense), and in the end the test system was limited to quasistatic analysis or "pure-moment" load control schemes.

\section{Biomechanical Testing Protocols}

Although a variety of different testing methods have been used to study cervical spine mechanics, the two most common ones are load and displacement control. ${ }^{18}$ Under load control, a pure or constant moment is incrementally applied to the spine and the spine is typically loaded in one plane of motion at a time (that is, sagittal, frontal, or transverse). Under displacement control, the translational and rotation motions of the vertebrae are controlled. Our biomechanical testing apparatus can be controlled under load, displacement, or a hybrid control (displacement with force feedback). In this study the displacement of the spine was controlled and, by using custom fixtures, a "moment distribution" was induced throughout the spine.

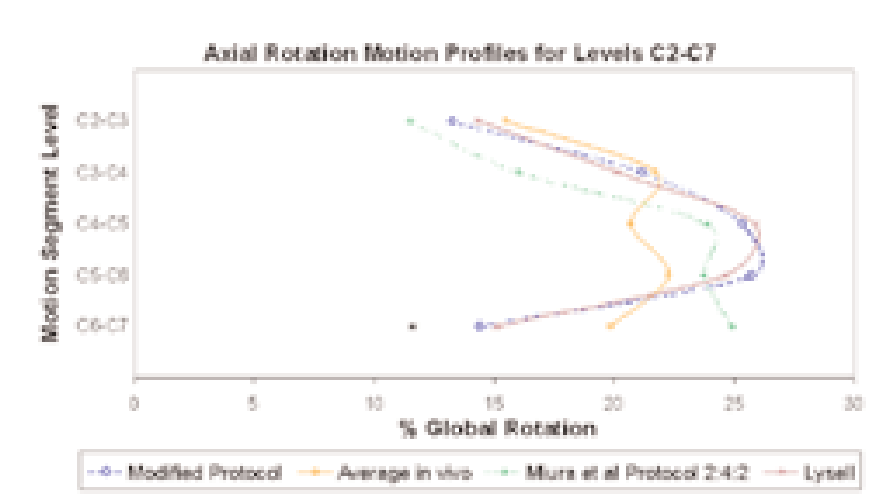

Fig. 14. Graph showing MSU contribution of global axial rotations for different in vitro testing methods compared with published in vivo data. Asterisk denotes significant difference between modified and pure-moment methods $(\mathrm{p}<0.01)$. 
Other parameters were also monitored to establish the upper motion limit; these included the applied moment and load. A limit value was assigned to each parameter and the test was stopped if any limit checks were exceeded. This arrangement is vastly different from and superior to pure-moment methods, in which there is no limit check on the resultant motion. Furthermore, pure-moment protocols typically use moment values in the range of 1 to 2 $\mathrm{Nm}$. When analyzing cervical spine instrumentation, however, this load level may not be sufficient to induce measurable differences in the motion response.

The question arises as to which testing method better replicates the in vivo motion behavior of the cervical spine. Miura, et al., ${ }^{24}$ recently described a method to simulate in vivo cervical spine kinematics by using a preload and pure-moment protocol. The technique of a follower load was used in conjunction with a pure moment. A critical detail of the follower load concept is the ability to pass a compressive load through the IARs of each MSU. In the aforementioned study, the IAR was placed near the lateral masses and remained fixed for the flexion and extension tests. This IAR position was based, however, on three cited studies, $, 2,1433$ in none of which was performed an analysis of the propagation of error associated with the theoretical calculation itself, nor were the IAR centers determined over small ranges of motion that typically occur between two adjacent MSUs (that is, 2-3 increments). We have previously shown that the error in calculating the location of the IAR can be large (as high as \pm $10 \mathrm{~mm}$ ) for small angular changes $\left(2-3^{\circ}\right)$ and that the IAR position is significantly different in flexion from that in extension. ${ }^{11}$ Furthermore, the axis of rotation of the cervical spine varies between flexion and extension, but was constrained from moving to apply the compressive follower load. This arrangement may limit or alter the motion response.

To understand the differences in the MSU rotation patterns for the different loading conditions, only the mean values of the percent contributions of the MSU rotations were plotted in Figs. 8, 11, and 14. For flexion/extension loading, the motion response obtained using a pure-moment protocol with a follower load was found to be representative of in vivo spine studies. ${ }^{13,15,19,22,29,30}$ Nevertheless, the combined mean flexion/extension rotational values did not always follow the in vivo pattern and in some instances went in the opposite direction or remained constant across multiple MSU levels (see Fig. 7) at the region where the predominant amount of motion occurs in the cervical spine (that is, C4-5 and C5-6). The trend in these authors' data indicates that if the sample size were increased, significant differences would exist between their in vitro data and the in vivo findings.

The follower load concept was developed by Patwardhan, et al. ${ }^{27}$ to allow the intact spine to withstand greater compressive loads without buckling. A compressive load was applied along the bending axis of the spine to simulate the net resultant action of muscles on either side of the spine. The follower load has been successfully used to demonstrate how the intact multisegmental spine can withstand large compressive loads without buckling. Nevertheless, use of the follower load to study the instrumented multiple-level cervical spine may artificially add more stability to the spine than occurs in vivo. ${ }^{5}$ Disc replacement or motion preservation devices may or may not have a fixed axis of rotation. The location of the follower load relative to the rotational axis directly affects how the device transfers load and maintains joint stability. Furthermore, although the follower load is traditionally applied to the flexion/extension plane, its load-transferring concept also affects how the load and motion respond in the transverse and frontal planes.

\section{CONCLUSIONS}

When evaluating the performance of disc arthroplasty or motion preservation devices, not only should the instrumented level be analyzed, but changes at the adjacent and remaining segments should be included in the analysis. Therefore, the preferred testing protocol is one that most closely follows the in vivo pattern for all segments of the cervical spine. An improved biomechanical testing protocol was developed that better replicated the physiological motion response of the cervical spine. In contrast, application of pure-moment loading methods did not replicate the physiological response and is less suited for evaluating disc arthroplasty and nonfusion hardware.

\section{Acknowledgments}

Tissue procurement was coordinated through the Medical Education and Research Institute, Memphis, Tennessee. We thank Henry Bonnin for assistance with data processing and manuscript preparation and Eve Blair, Thomas Jansen, Jaclyn Kuspiel, Brian Morrow, John Schwab, Amanda Thomas, and Keith Vossel for technical assistance with the experimental tests.

\section{References}

1. Amevo B, Macintosh JE, Worth D, et al: Instantaneous axes of rotation of the typical cervical motion segment: I. an empirical study of technical errors. Clin Biomech 6:31-37, 1991

2. Amevo B, Worth D, Bogduk N: Instantaneous axes of rotation of the typical cervical motion segments: a study in normal volunteers. Clin Biomech 6:111-117, 1991

3. Chen J: Development of a Flexible Biomechanical Testing Apparatus. MS Thesis. Memphis: University of TennesseeMemphis, 1996

4. Cherubino P, Benazzo F, Borromeo U, et al: Degenerative arthritis of adjacent spinal joints following anterior cervical spinal fusion: clinicoradiologic and statistical correlations. Ital J Orthop Traumatol 1990;16:533-543

5. Cripton PA, Miura T, Panjabi MM: The effects of physiologic compressive preload on the in vitro flexibility of the lower cervical spine, in Proceedings of the 2001 Cervical Spine Research Society. Rosemont, IL: CSRS, 2001, pp 214-215 (Abstract)

6. Cummins BH, Robertson JT, Gill SS: Surgical experience with an implanted artificial cervical joint. J Neurosurg 88:943-948, 1998

7. DiAngelo DJ, Faber HB, Dull ST, et al: Development of an in vitro experimental protocol to study the extensional mechanics of the cervical spine, in Simon B (ed): 1997 Advances in Bioengineering. New York: ASME, 1997, Vol 36, pp 211-212

8. DiAngelo DJ, Foley KT, Vossel KA, et al: Anterior cervical plating reverses load transfer through multilevel strut-grafts. Spine 25:783-795, 2000

9. DiAngelo DJ, Jansen TH, Eckstein EC, et al: Measurements for the in vitro failure of multi-level instrumented cervical spine, in 
Simon B (ed): 1997 Advances in Bioengineering. New York: ASME, 1997, Vol 36, pp 223-224

10. DiAngelo DJ, Robertson JT, Metcalf NH, et al: Biomechanical testing of an artificial cervical joint and an anterior cervical plate. J Spinal Disord Tech 16:314-323, 2003

11. DiAngelo DJ, Vossel KA, Foley KT: The instant axis of rotation of the cervical spine in flexion and extension, in Proceedings of the 2000 Cervical Spine Research Society. Rosemont, IL: CSRS, 2000, pp 203-204 (Abstract)

12. DiAngelo DJ, Vossel KA, Jansen TH: A multi-body optical measurement system for the study of human joint motion, in Yoganathan AP (ed): 1998 Advances in Bioengineering. New York: ASME, 1998, Vol 39, pp 195-196

13. Dvorak J, Froehlich D, Penning L, et al: Functional radiographic diagnosis of the cervical spine: flexion/extension. Spine 13: 748-755, 1988

14. Dvorak J, Panjabi MM, Novotny JE, et al: In vivo flexion/ extension of the normal cervical spine. J Orthop Res 9: 828-834, 1991

15. Dvorak J, Panjabi MM, Grob D, et al: Clinical validation of functional flexion/extension radiographs of the cervical spine. Spine 18:120-127, 1993

16. Foley KT, DiAngelo DJ, Rampersaud YR, et al: The in vitro effects of instrumentation on multilevel cervical strut-graft mechanics. Spine 24:2366-2376, 1999

17. Gilbertson LG, Doehring TC, Livesay GA, et al: Improvement of accuracy in a high-capacity, six degree-of-freedom load cell: application to robotic testing of musculoskeletal joints. Ann Biomed Eng 27:839-843, 1999

18. Goel VK, Wilder DG, Pope MH, et al: Biomechanical testing of the spine. Load-controlled versus displacement-controlled analysis. Spine 20:2354-2357, 1995

19. Holmes A, Wang C, Han Z.H, et al: The range and nature of flexion-extension motion in the cervical spine. Spine 19: 2505-2510, 1994

20. James KS, Wenger KH, Schlegel JD, et al: Biomechanical evaluation of the stability of thoracolumbar burst fractures. Spine 19:1731-1740, 1994

21. Kunz DN, McCabe RP, Zdeblick TA, et al: A multi-degree of freedom system for biomechanical testing. J Biomech Eng 116:371-373, 1994

22. Lind B, Sihlbom H, Nordwall A, et al: Normal range of motion of the cervical spine. Arch Phys Med Rehabil 70:692-695, 1989

23. Lysell E: Motion in the cervical spine. An experimental study on autopsy specimens. Acta Orthop Scand (Suppl 123):1-61, 1969

24. Miura T, Panjabi MM, Cripton PA: A method to simulate in vivo cervical spine kinematics using a compressive preload. Spine 27:43-48, 2002

25. Miura T, Panjabi MM, Cripton PA: Stability of three strut-graft constructs for multi-level cervical corpectomy, in Proceedings of the 2001 Cervical Spine Research Society. Rosemont, IL: CSRS, 2001, pp 55-56 (Abstract)

26. Parsons JR, Zimmerman MC, Lee CK, et al: Examination of the failure mode of several cervical spine fixation devices, in Langrana NA, Friedman MH, Grood ES (eds): 1993 Bioengineering Conference. New York: ASME, 1993, Vol 24, pp 175-178

27. Patwardhan AG, Havey RM, Ghanayem AJ, et al: Load-carrying capacity of the human cervical spine in compression is increased under a follower load. Spine 25:1548-1554, 2000

28. Pelker RR, Duranceau JS, Panjabi MM: Cervical spine stabilization. A three-dimensional, biomechanical evaluation of rotational stability, strength, and failure mechanisms. Spine 16: 117-122, 1991

29. Penning L: Normal movements of the cervical spine. AJR 130: 317-326, 1978

30. Ordway NR, Seymour RJ, Donelson RG, et al: Cervical flexion, extension, protrusion, and retraction. A radiographic segmental analysis. Spine 24:240-247, 1999

31. Shea M, Edwards WT, White AA, et al: Variations of stiffness and strength along the human cervical spine. J Biomech 24: 95-107, 1991

32. Smith SA, Lindsey RW, Doherty BJ, et al: An in vitro biomechanical comparison of the orosco and ao locking plates for anterior cervical spine fixation. J Spinal Disord 8:220-223, 1995

33. van Mameren H, Sanches H, Beursgens J, et al, Cervical spine motion in the sagittal plane. II. Position of segmental averaged instantaneous centers of rotation-a cineradiographic study. Spine 17:467-474, 1992

34. Weinhoffer SL, Guyer RD, Herbert M, et al: Intradiscal pressure measurements above an instrumented fusion. A cadaveric study. Spine 20:526-531, 1995

35. White AA III, Panjabi MM: Clinical Biomechanics of the Spine, ed 2. Philadelphia: Lippincott Williams \& Wilkins, 1990

36. Wilke HJ, Wolf $\mathrm{S}$, Claes LE, et al: Influence of varying muscle forces on lumbar intradiscal pressure: an in vitro study. J Biomech 29:549-555, 1996

Manuscript received June 29, 2004.

Accepted in final form August 16, 2004.

Address reprint requests to: Denis J. DiAngelo, Ph.D., Department of Biomedical Engineering, The University of Tennessee Health Science Center, 920 Madison Avenue, Suite 1005, Memphis, Tennessee 38163. 